\title{
Industrial Applications of Plasma Focus Radiation
}

\author{
C. Moreno, M. Vénere, R. Barbuzza, M. Del Fresno, R. Ramos, \\ H. Bruzzone, Florido P. J. González, and A. Clausse \\ Interinstitutional Program of Dense Magnetized Plasmas, \\ CNEA-CONICET-CIC-INFIP-UNMP-UNICEN, Argentina
}

Received on 3 July, 2001

\begin{abstract}
Applications of a small-chamber Plasma Focus used as portable radiation generator is presented. The device was designed to maximize the fluence. The mean neutron yield was $3 \times 10^{8}$ neutrons of $2.45 \mathrm{MeV}$ per shot, corresponding to a $10^{6}$ neutrons $/ \mathrm{cm}^{2}$ fluence on the external surface of the chamber. A technique to detect the presence of water in the neighborhood of a compact Plasma Focus is presented. The measuring system is composed by two neutron detectors operated simultaneously on every shot. The first detector is used to register the PF neutron yield in each shot; whereas the other one was designed for detecting neutrons scattered by the blanket. The results indicate that the system is able to detect water contents of few percents in volume. The correlation of the counts recorded by the detectors located at different positions was mapped with the water distribution around the neutron source. The complete detecting system is very simple and inexpensive. Among many other potential applications, the technique is specially suited for soil humidity prospection. $\mathrm{X}$ rays radiation emitted by the compact Plasma Focus operated in Deuterium has been used for introspective radiographic imaging of metallic objects. The samples were located about $1 \mathrm{~m}$ away from the PF chamber wall. High-sensitivity, fast-response commercial radiographic film was used as $\mathrm{x}$-ray detector. A set of experimental images is presented demonstrating a very high penetration power of the x-ray beam. Among many other applications, the presented technique is specially suited for introspective visualization of pieces manufactured on metal. Radiographic projections of a stainless steel BNC elbow taken at 8 different angles were processed to reconstruct transversal cuts of the piece. A computer technique for 3D reconstructions was combined with radiographic images of objects X-rayed with a compact plasma focus. The technique is able to automatically determine the position of the rotation axis, reconstruct the 3D-attenuation map, and display inner cuts. The system was demonstrated in introspective tomographic imaging of a stainless steel BNC elbow.
\end{abstract}

\section{Introduction}

Plasma Focus (PF) devices flourished in the 70's and 80 's as nuclear fusion devices based in the pinch phenomenon occurring during the path of high electric currents through the working gas. The operation of PF has been extensively studied by research laboratories around the world, where several PF configurations has been developed over the years aiming to increase the neutron emission [1-4]. Currently, PF pulsors are among the cheapest available neutron generators, with unique features of extremely short pulses (hundreds of ns) that suit them for a number of interesting applications. There are also interesting possibilities to take advantage of $\mathrm{x}$-rays (1-100 keV), electron and ion beams emitted during PF shots.

The plasma-focus phenomenon occurs at the open end of coaxial electrodes when an intense electrical discharge between them is induced by external means. The coaxial electrodes are located inside a vacuum chamber filled with deuterium gas at low pressure. A charged capacitor bank is connected to the closed end of the electrodes through a switch. After closing the switch, a gas discharge starts in the gap between the electrodes forming an umbrella-like plasma layer. The azimuthal magnetic field located in the toroidal volume enclosed by the current, produces a Lorentz force that pushes the sheath toward the open end of the electrodes. The run-down of the current sheath is a sweeping supersonic shock that propagates collecting the gas particles ahead of the front. On its arrival at the open end (some $\mu \mathrm{s}$ after triggering), the magnetic field starts to contract, 
accelerating the plasma toward the axis. Finally, the sheath clashes on the axis in the form of a small dense plasma cylinder (focus). The lifetime of the focus is about $300 \mathrm{~ns}$.

The emitted neutrons can be applied to perform radiographs [5] and substance analysis, taking advantage of the penetration and activation properties of neutral radiation [6]. Likely, the intense x-ray pulses produced by focalized electron bremstralung are excellent candidates for radiography of moving and soft objects and for microelectronic lithography [7].

Had small portable PF devices been available, the added value of the emissions would substantially increase, for larger fluences can be provided in wider domains of applications. However, due to the strong interaction between the hot plasma and the vacuum chamber, the electrode housing is usually big, leaving room for the plasma blast. The main trouble with having the electrodes too close to the container wall is the gas contamination with impurities, which conspires against performance and regularity of the emissions [8].

Along this article, applications of the compact PF device, pulsing at one shot per minute is presented. The associated $\mathrm{x}$-rays emissions were applied to obtain three-dimensional introspective images of small metallic components, and the emitted neutrons were used to detect water by neutron inelastic scattering.

\section{The plasma focus GN1}

The Plasma-Focus GN1 is a compact version of a Mather-type machine. Fig. 1 shows a diagram of the vacuum chamber and the electrodes. The anode is an electrolytic-copper cylinder, $38 \mathrm{~mm}$ diameter, $1.5 \mathrm{~mm}$ thick, $87 \mathrm{~mm}$ long. The cathode is formed by 12 bronze bars, $3 \mathrm{~mm}$ diameter, $100 \mathrm{~mm}$ long, cylindrically placed, and welded at the end to a bronze ring $72 \mathrm{~mm}$ diameter. The container is a stainless-steel cylinder $157 \mathrm{~mm}$ long, $96 \mathrm{~mm}$ diameter with a lateral NW25 vacuum port for pumping and gas loading. The insulator is a Pyrex glass cylinder $35 \mathrm{~mm}$ long and $4 \mathrm{~mm}$ thick.

Using a mechanical pump and an oil diffuser, base pressures down to $10^{-8}$ mbar can be reached. The external circuit is a capacitor bank, divided in three discharging modules; each of them composed by five Maxwell type 31161 condensers. The total capacitance is $10.5 \mu \mathrm{F}$ and the charging voltage is $30 \mathrm{kV}$. The three modules are fired simultaneously and peak currents of $350 \mathrm{kA}$ are attained in a quarter of period $(\sim 1.1 \mu \mathrm{s})$. The system operates between 1 to 8 mbar of Deuterium.

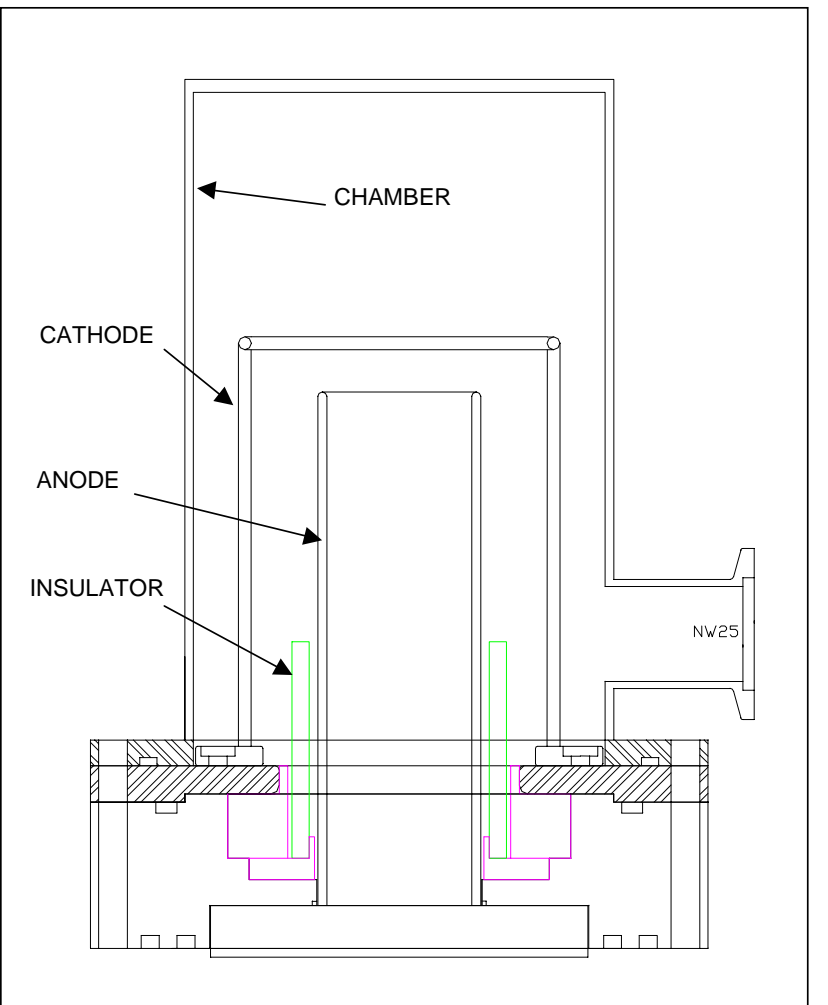

Figure 1. Schematics of GN1 chamber (sizes are detailed in the text).

After each shot, the filling pressure increases about 0.05 mbar due to the release of impurities from the chamber, electrodes and insulator walls. Consequently, the chamber is pumped down (mechanically) after each shot in order to assure constant pressure conditions. The maximum shot frequency was one shot per minute, limited by the charger. Under these conditions, the frontal wall temperature (top in Fig. 1) increases about $20{ }^{\circ} \mathrm{C}$ over the ambient temperature after 30 shots, cooled passively by air natural convection and heat conduction through the metallic structure. The working gas is renewed after 10 shots.

Upon commissioning, the equipment was tested in a series of 1000 shots at different load pressures. The time derivative of the current flowing to the anode, $d I / d t$, and the voltage across the electrodes, $V$, were monitored for each shot by a Rogowski coil and a resistive voltage divider, and were registered using a $500 \mathrm{MHz}$, $1 \mathrm{Gs} / \mathrm{s}$ digitizing oscilloscope. Fig. 2 shows typical signals for filling pressures of $2 ; 4$ and 6 mbar. Very intense voltage spikes $(\sim 120 \mathrm{kV})$ are obtained at the time where the maximum compression takes place, thus indicating good focusing. Such peaks impose severe design conditions on the insulator, which should be considerably thick in order to stand the stress ( $2 \mathrm{~mm}$ of Pyrex glass is destroyed with few discharges). It was observed in Fig. 2 that the focus occurs later for higher pressures. Fig. 3 shows the average dependence of the focus timing with the deuterium pressure. The dimensions of the electrodes were determined using a computer aided 
design system which is based in a thermonuclear PF model [9]. The continuous trace is the focussing time derived from an analytical snowplow description coupled with an isoentropic plasma compression model.
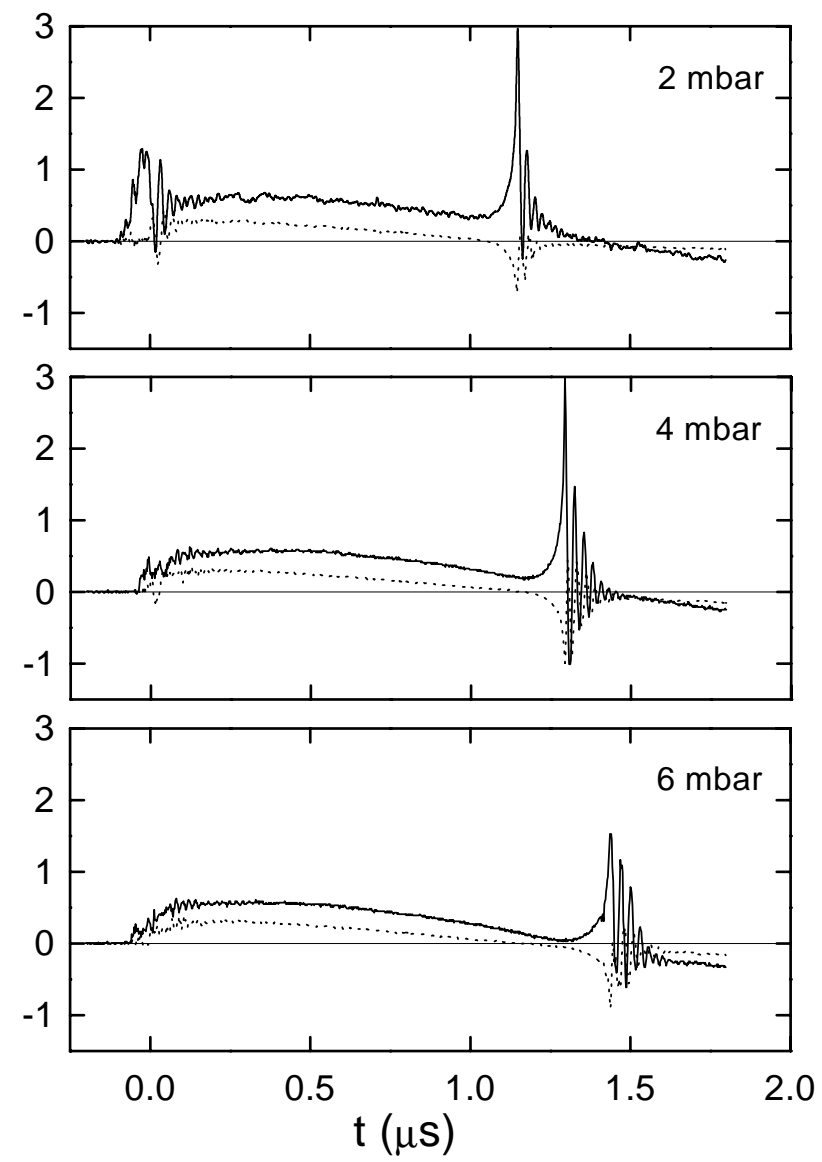

Figure 2. Signals proportional to the voltage across electrodes (solid line) and current time derivative (dotted line). The signals units are Volts measured in a Rogowski coil and a voltage divider respectively.

The time integrated neutron yield was also measured for each shot by silver activation response as a function of pressure. The neutron detector was placed at $60 \mathrm{~cm}$ from the focus in a line perpendicular to the axis. The sensitive area was $900 \mathrm{~cm}^{2}$. The neutron measuring system was calibrated by comparison against TLD detectors. Fig. 4 shows the dependence of the average neutron yield per pulse with the filling pressure. The continuous trace on Fig. 4 corresponds to same model of Fig. 3. The optimum average yield, $3 \times 10^{8}$ neutrons per shot, occurs at 4 mbar. This yield corresponds to $10^{6} \mathrm{n} / \mathrm{cm}^{2}$ per shot in the external frontal wall.

Different insulator lengths were tested in preliminary series of measurements aimed to investigate each design performance. It was found that variations of $5 \mathrm{~mm}$ in that length lead to noticeable degradation of the neutron yield at all the explored pressures.

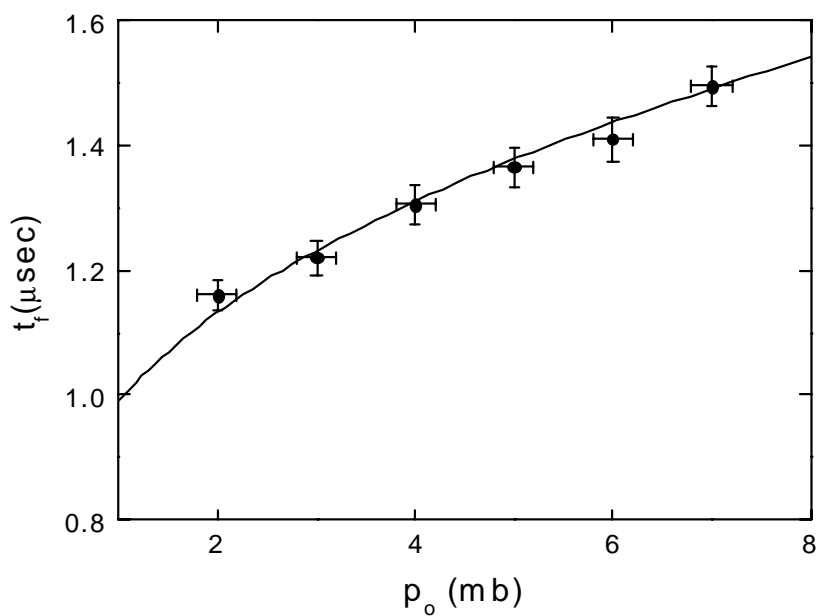

Figure 3. Variation of the focusing timing with filling pressure. The line corresponds to predictions of a snowplow model [9].

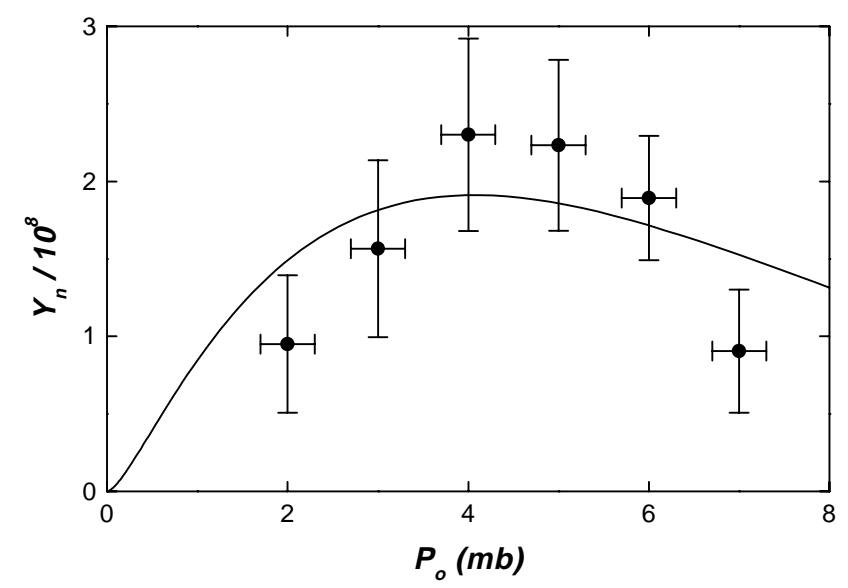

Figure 4. Neutrons yield at $90^{\circ}$ off axis as function of filling pressure. The line corresponds to predictions of a snowplow model [9].

\section{Defectoscopy}

PF can be advantageously used as high-intensity short-duration x-ray sources. Due to the very fast plasma compression attained in these devices, particularly intense soft and hard x-rays pulses are emitted. High repetition rate small $\mathrm{PF}$ are currently used for SXR lithography [10]. High-Z working gases are used in these applications to enhance the x-ray yield at reduced wavelengths [5]. We decided to use the compact PF operated in Deuterium for making non-conventional radiographs. Our aim is to find new application fields for the PF as x-ray source, and to allow for the possibility of obtaining, in a future experiment, a simultaneous radiographic and neutrographic image of the same object. 
The samples to be imaged were placed outside the stainless steel chamber, on the electrodes symmetry axis, and $83.5 \mathrm{~cm}$ away from the focussing region (Fig. 5). Commercial radiographic film, Curix ST-G2 from AGFA was used together with AGFA suggested developer and fixer for this film. No special procedures were needed other than those recommended by the supplier, to manipulate and develop the films.

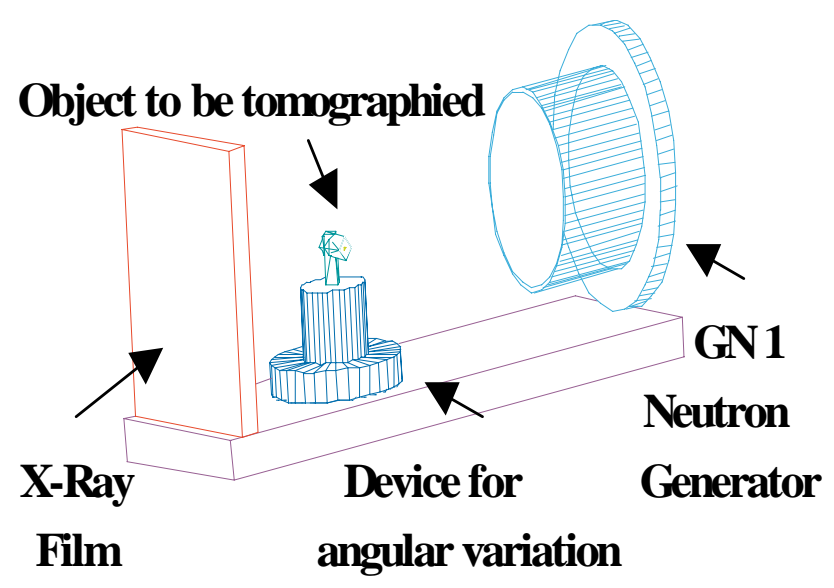

Figure 5. Setup of the tomographic technique.

A stainless steel BNC elbow was used as a sample. All the shots were made at filling pressures of 4-5 mbar pure deuterium. The sample was mounted on a small acrylic platform that rotates to allow for taking images at different viewing angles. The rotating axis was set vertically and 8 viewing angles were used: $0,30,60,75$, 90, 105, 120, and 150 degrees. The axis was marked with a sharp metallic needle.

Fig. 7 shows the set of radiographs of the piece at different angles. It can be seen that, even controlling carefully the operation, there are always differences in brightness and focalization. Therefore, in order to be useful in a tomographic system, we should be able to reconstruct inner cuts processing imperfect projections.

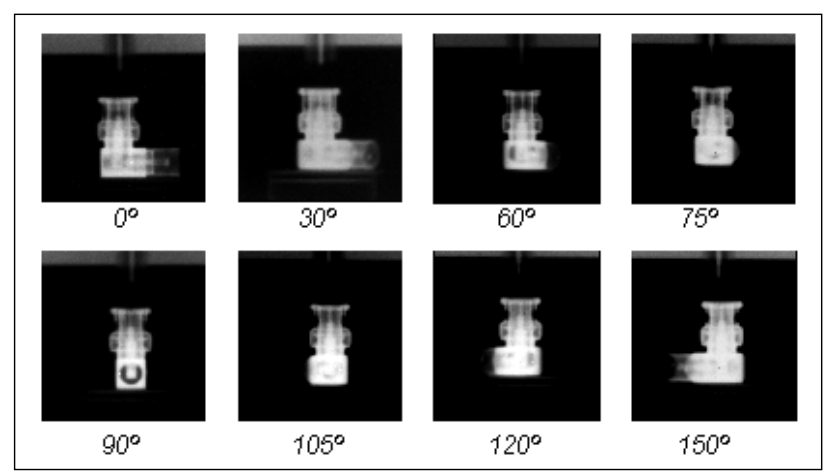

Figure 6. Angular radiographs of a BNC.

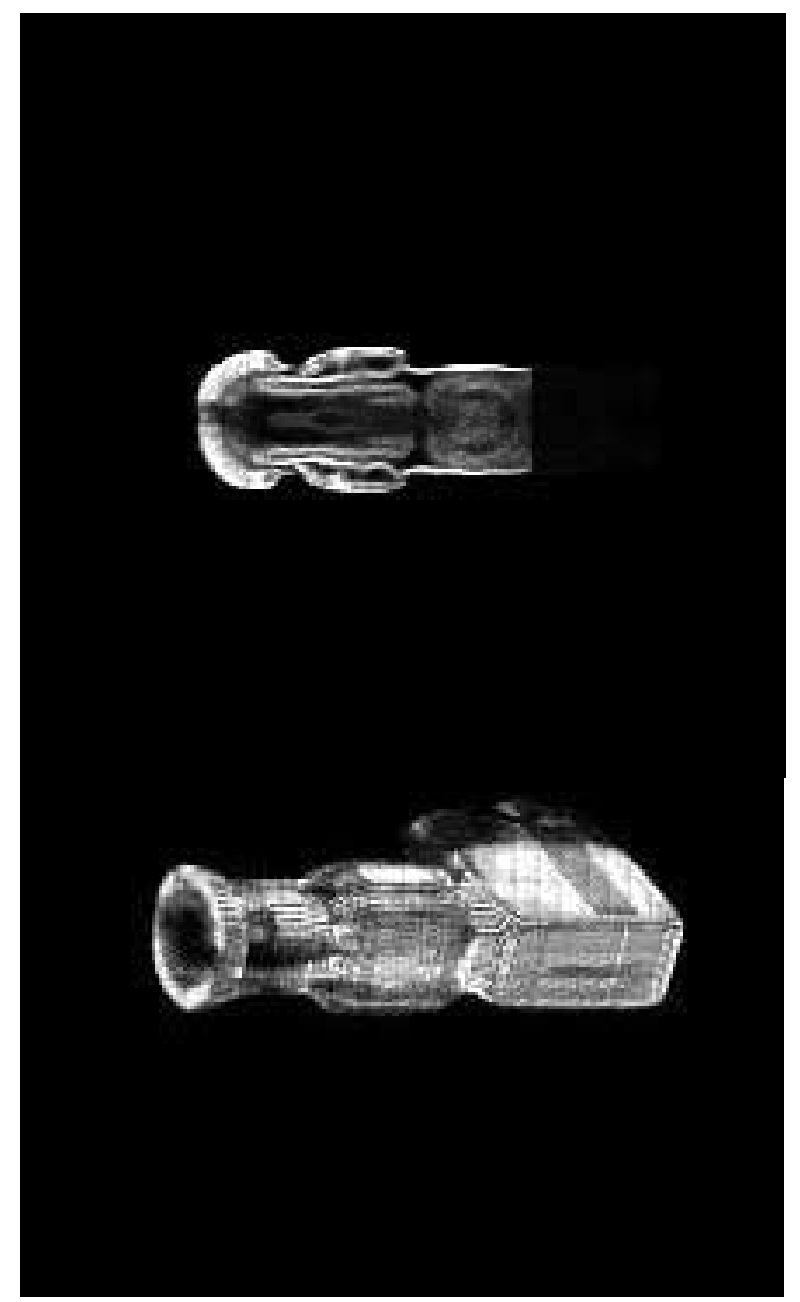

Figure 7. Tomographic reconstructions using the radiographs shown in Fig. 6.

The Montecarlo method is a flexible technique than can to handle quality differences of the information input. Basically, Montecarlo tomographic reconstruction is a stochastic searching process, where the computer bounces randomly in the set of all possible digital 3D images (called instances), guided with a selection criterion that ensures the convergence toward the actual inner attenuation field. The reconstruction algorithm is based on the comparison of the actual radiographs with the projections that would produce the instance image. The general procedure is as follows:

Starting with an initial guess instance, modify slightly the tone of a pixel chosen at random.

Calculate the projections of the instance in every direction.

Calculate an error indicator averaging the square deviations of the instance projections respect to the actual projections.

If the error of the new instance is lower than the previous one, the modification is accepted. 
The method was implemented using object oriented programming, in visual $\mathrm{C}^{++}$. A visualization system completes the tool, allowing the fast inspection of inner cuts of the attenuation field. The hardware requirement is just a Pentium personal computer, and the system runs in MS Windows environment. Fig. 7 shows the display of different cuts of the $\mathrm{BNC}$ on the control panel of the application. The visualization of the inner cuts shows details down to $0.3-\mathrm{mm}$ resolution.

\section{Neutron echography}

Neutrons can be used for detecting Hydrogen in metals or for detecting substances by activation analysis [11]. Either huge fission reactors or powerful deuteron accelerators are used in these applications as neutron sources, which share the disadvantages of being nonportable and very expensive. On the contrary, substance interrogation system should be portable, reliable, economically competitive, and minimize the environmental impact. PF devices filled with deuterium gas, complemented with a couple of neutronic detectors are a valid alternative, as they fulfill all the requirements mentioned before.

We conducted an experiment to detect water or other substances containing low Z elements, by neutron inelastic scattering. The method is based on the same principle of the sonar or the echographs, registering the neutrons scattered by the interrogated substance, comparing the detector response when the interrogated substance is absent.

The measuring system is composed by a neutron pulsed generator and two silver activation detectors operated simultaneously on every shot. The first detector is used to register the PF neutron yield; whereas the other one is used to detect neutrons scattered by a water blanket. The compact PF device filled with deuterium was used to provide neutron pulses. The basic idea of the method is to use a suitable detector to register the neutrons scattered by the interrogated substance (if present) and to contrast the detector response when the interrogated substance is absent. Shots were made in presence of the interrogated substance, then the procedure was repeated after removing the substance, and the neutrons registered in both situations were compared.

Fig. 8 shows the experimental setup. A standard silver activation counter placed side-on, $60 \mathrm{~cm}$ away from the PF chamber was used as the reference detector to take the shot to shot variation of the neutron yield into account. This detector was covered with paraffin to moderate the fast neutrons. The second detector is composed by three Geigers, each of them covered by a $0.3 \mathrm{~mm}$ thick silver foil and placed inside a metallic can (10 $\mathrm{cm}$ in diameter and $13 \mathrm{~cm}$ in length). Some packaging plastic was used to keep the Geigers assembly in position inside the can. This detector was located side-on, approximately at $30 \mathrm{~cm}$ from the PF chamber.

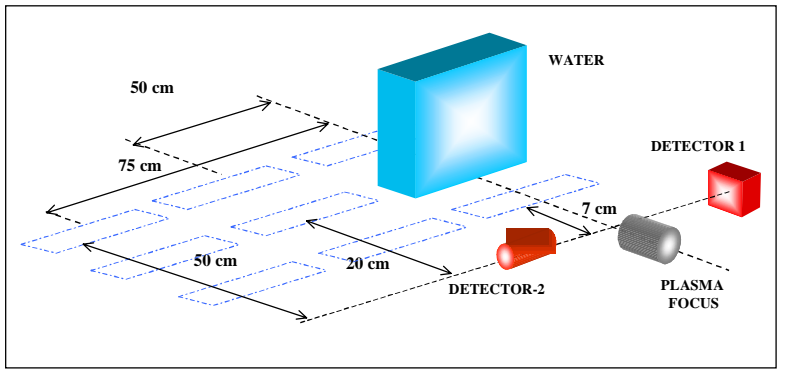

Figure 8. Neutron echo interrogation setup.

140 liters of water were arranged in a wall of 11 plastic containers. The water wall was put in different positions respect of the detector systems. For every configuration the $\mathrm{PF}$ was shot about fifteen times recording the counts measured in each detector.

Fig. 9 shows the results obtained along the PF axis. Each point in the graphic corresponds to one shot, the counts of each detector being the coordinates of the plot. The relative counting of the moderated detector increases as the water gets closer to the detection system. This effect can be attributed to the change in the solid angle offered by the water. The counts collected by the scattered neutrons detector when no water is present, correspond to neutrons reflected by the laboratory building. The slope of the least-squares lines corresponding to each position can be used to characterize the sensitivity of the system. Fig. 10 shows the contour map of the relative counting slope. The coordinates correspond to the location of the water wall respect to the $\mathrm{PF}$ head. The $\mathrm{x}$ - and $\mathrm{y}$-coordinates are measured along lines perpendicular and parallel to the PF symmetry axis respectively. It can be seen that the relative counting decreases faster when the water is moved away along the symmetry axis.

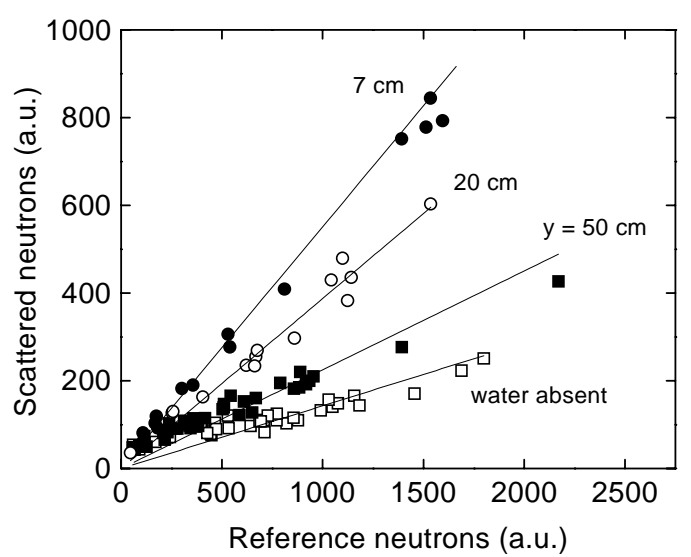

Figure 9. Neutronic reflection in along the PF axis. 


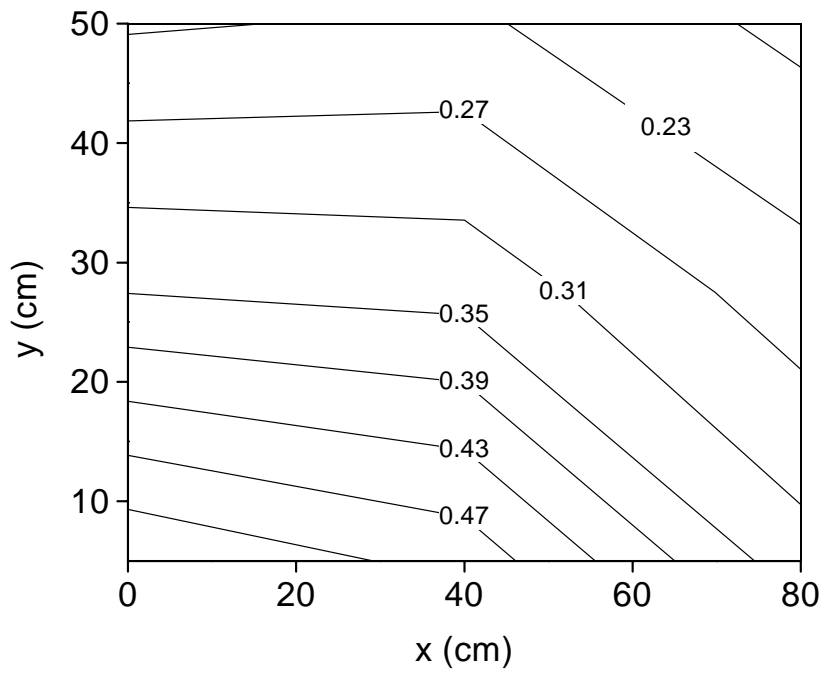

Figure 10. Contour map of the relative neutron counting. Every $(\mathrm{x}, \mathrm{y})$ coordinate represent the position of the water.

\section{Conclusions}

Two feasibility studies of industrial applications of the $\mathrm{X}$ and neutron radiation produced by a compact Plasma Focus were presented.

A technique to detect the presence of water was developed using two neutron detectors operated simultaneously on every shot. The results indicate that the system is able to detect water contents of few percents in volume. The complete detecting system is very simple and inexpensive. Among many other potential applications, the technique is specially suited for soil humidity prospection.

On the other hand, X-rays from the PF has been used for introspective radiographic imaging of metallic objects. The technique is specially suited for introspective visualization of pieces manufactured on metal. Radiographic projections of a stainless steel BNC elbow taken at 8 different angles were processed to reconstruct transversal cuts of the piece. A computer technique for $3 \mathrm{D}$ reconstructions was combined with radiographic images of objects X-rayed with a compact plasma focus. The technique is able to automatically determine the position of the rotation axis, reconstruct the 3Dattenuation map, and display inner cuts.

\section{References}

[1] A. Bernard, Nuclear Instruments Methods, 145, 191 (1977).

[2] H. Conrads, Proc. $3^{\text {rd }}$ Latinamerican Worshop Plasma Phys., Santiago, Chile, July 18-29 (1989).

[3] A. Serban and S. Lee. Experiments on speed-enhanced neutron yield from a plasma focus. J. Plasma Physics, 60, part 1, 3-15 (1998).

[4] G. Decker, R. Wienecke, Physica 82C, 155-164 (1976).

[5] M. Gibbons, W. Richards and K. Shields. Optimization of neutron tomography for rapid $\mathrm{H}$ concentration inspection of metal castings, LLNL Rep UCRL-JC129723, (1998).

[6] E. Hussein and E. Waller. Review of one-side approaches to radiographic imaging for detection of explosives and narcotics, Radiation Measurements, 29 (6), 581, (1998).

[7] S. Lee, V. Kudryashov, P. Lee, G. Zhang, A. Serban, M. Liu, X. Feng, S. Springham, T. Wong and C. Selvam. SXR Lithography Using a High Performance Plasma Focus Source, 1998 ICPP and 25 EPS Conf on Contr. Fusion and Plasma Physics, 22C, 2591 (1998).

[8] J. Gratton, M. Alabraba, A. Warmate and G. Giudice, Deterministic dynamics of Plasma Focus discharges. Chaos, Solitons \& Fractals, 3, 343 (1993).

[9] C. Moreno, H. Bruzzone, J. Martínez and A. Clausse. Conceptual engineering of plasma-based neutron pulsors. IEEE Transactions on Plasma Science, 28, 17351741 (2000).

[10] S. Lee, P. Lee, G. Zhang, X. Feng, V. Gribkov, M. Liu, A. Serban, and T. Wong. High Rep Rate High Performance Plasma Focus as a Powerful Radiation Source. IEEE Trans on Plasma Sci, 26, 1119, Aug (1998).

[11] F. Brooks, A. Buffler, M. Allie, K. Bharuth-Ram, M. Nchodu and B. Simpson, Determination of HCNO concentrations by fast neutron scattering analysis, Nuclear Instruments and Methods Sect. A (410) 2, 319-328 (1998). 